\title{
Video Article \\ Modeling Stroke in Mice - Middle Cerebral Artery Occlusion with the Filament Model
}

\author{
Odilo Engel ${ }^{1}$, Sabine Kolodziej ${ }^{1}$, Ulrich Dirnagl ${ }^{1}$, Vincent Prinz ${ }^{1}$ \\ ${ }^{1}$ Department for Experimental Neurology, Center for Stroke Research Berlin, Charité Universitätsmedizin
}

Correspondence to: Ulrich Dirnagl at ulrich.dirnagl@charite.de

URL: https://www.jove.com/video/2423

DOI: doi:10.3791/2423

Keywords: Medicine, Issue 47, Stroke, middle cerebral artery occlusion, MCAo, animal model, mouse, techniques

Date Published: 1/6/2011

Citation: Engel, O., Kolodziej, S., Dirnagl, U., Prinz, V. Modeling Stroke in Mice - Middle Cerebral Artery Occlusion with the Filament Model. J. Vis. Exp. (47), e2423, doi:10.3791/2423 (2011).

\section{Abstract}

Stroke is among the most frequent causes of death and adult disability, especially in highly developed countries. However, treatment options to date are very limited. To meet the need for novel therapeutic approaches, experimental stroke research frequently employs rodent models of focal cerebral ischaemia. Most researchers use permanent or transient occlusion of the middle cerebral artery (MCA) in mice or rats.

Proximal occlusion of the middle cerebral artery (MCA) via the intraluminal suture technique (so called filament or suture model) is probably the most frequently used model in experimental stroke research. The intraluminal MCAO model offers the advantage of inducing reproducible transient or permanent ischaemia of the MCA territory in a relatively non-invasive manner. Intraluminal approaches interrupt the blood flow of the entire territory of this artery. Filament occlusion thus arrests flow proximal to the lenticulo-striate arteries, which supply the basal ganglia. Filament occlusion of the MCA results in reproducible lesions in the cortex and striatum and can be either permanent or transient. In contrast, models inducing distal (to the branching of the lenticulo-striate arteries) MCA occlusion typically spare the striatum and primarily involve the neocortex. In addition these models do require craniectomy. In the model demonstrated in this article, a silicon coated filament is introduced into the common carotid artery and advanced along the internal carotid artery into the Circle of Willis, where it blocks the origin of the middle cerebral artery. In patients, occlusions of the middle cerebral artery are among the most common causes of ischaemic stroke. Since varying ischemic intervals can be chosen freely in this model depending on the time point of reperfusion, ischaemic lesions with varying degrees of severity can be produced. Reperfusion by removal of the occluding filament at least partially models the restoration of blood flow after spontaneous or therapeutic (tPA) lysis of a thromboembolic clot in humans.

In this video we will present the basic technique as well as the major pitfalls and confounders which may limit the predictive value of this model.

\section{Video Link}

The video component of this article can be found at https://www.jove.com/video/2423/

\section{Protocol}

To guarantee high quality and reproducibility, we recommend the use of Standard Operating Procedures (SOP). In this video, published SOPs as developed and used in our laboratory are applied. ${ }^{1}$

\section{Middle Cerebral Artery Occlusion}

1. Mice are anaesthetized with an appropriate anaesthetic regime in consult with veterinary staff. (E.g. induction with $1.5-2 \%$ Isoflurane and maintainance with $1.0-1.5 \%$ Isoflurane in $2 / 3 \mathrm{~N} 2 \mathrm{O}$ and $1 / 3 \mathrm{O} 2$ using a vaporizer).

1. Body temperature of the mice is maintained at $36.5^{\circ} \mathrm{C} \pm 0.5^{\circ} \mathrm{C}$ during surgery with a heating plate. A feedback controlled heating pad, which warms according to the rectal temperature of the mouse, is highly recommended.

2. Disinfect the skin and surrounding fur with an appropriate agent (e.g. $70 \%$ ethyl alcohol) and dry it afterwards.

2. A midline neck incision is made and the soft tissues are pulled apart.

3. The left common carotid artery (LCCA) is carefully dissected free from the surrounding nerves (without harming the vagal nerve) and a ligature is made using 6.0/7.0 string. 5.0 string can also be used.

4. The left external carotid artery (LECA) is then separated and a second knot is made.

5. Next, the left internal carotid artery (LICA) is isolated and a knot is prepared with a 6.0 filament.

6. After obtaining good view of the left internal carotid artery (LICA) and the left pterygopalatine artery (LPA), both arteries are clipped, using a microvascular clip.

7. A small hole is cut in the LCCA before it bifurcates to the LECA and the LICA. A monofilament made of 8.0 nylon coated with silicon hardener mixture (see below) is then introduced into the LICA, until it stops at the clip. Attention has to be paid not to enter the occipital artery. (Figure 1) 
8. The clipped arteries are opened while the filament is inserted into the LICA to occlude the origin of the LMCA in the circle of Willis.

9. The third knot on the LICA is closed to fix the filament in position.

10. The mice receive saline $0.5 \mathrm{~mL}$ subcutaneously as volume replenishment. For pain relief, Lidocaine gel is topically applied in the wound.

11. If reperfusion is intended, mice stay for $30-90$ min occlusion in a heated cage, the wound could be closed with a small suture clip. Afterwards, a second anaesthesia is performed, the third knot on the ICA is momentary opened and the filament withdrawn.

12. The remaining sutures are shortened and the skin is adapted with a surgical suture.

13. All animals receive a second volume replenishment as described above.

14. Animals are put in a heated cage for two hours to control for body temperature.

1. The animals must be checked daily after surgery for signs of discomfort. The mice could show some post surgical weight loss. They receive mashed food in a petri-dish placed on the floor to encourage eating. The food is replaced daily for seven days.

\section{Sham Operation}

1. For sham operations the filament is inserted to occlude LMCA and withdrawn immediately to allow instant reperfusion (1.8). The subsequent operation is identical to that performed on the animals undergoing cerebral ischaemia $(1.9-1.14)$ including a second anaesthesia.

\section{Preparation of the Filament}

1. Sterility of the filament should be considered. The use of sterilized equipment as well as an appropriate handling of the filament afterwards is a prerequisite for a sterile surgery. Disinfection of the filament is difficult, since many of the common sterilization methods may worsen the quality of the filament. However methods such as radiation, for example with UV light or $y$ rays, or chemical sterilization, for example with highly reactive gases such as ethylene oxide, are applicable.

2. 8.0 nylon filament is cut into lengths of $11 \mathrm{~mm}$ under the microscope

3. The filament tip must be coated completely and evenly over a length of $8 \mathrm{~mm}$ with a hardener mixture of Xantopren M Mucosa and Activator NF Optosil

\section{Representative Results}

Depending on the duration of blood flow restriction, different motor and behavioural deficits result. Both after 30 and 60 min of cerebral ischaemia, animals in most cases show decreased resistance to lateral push and circling due to disturbance in locomotion. Milder lesions manifest as a flexor position in the front limbs. These easily observable signs can be used as a basic score for the success of the operation. ${ }^{2}$

Morphologically the lesion can be assessed using either histology or Magnetic Resonance Imaging (MRI). Sixty minutes occlusion of the middle cerebral artery produces tissue pannecrosis in an area including both the striatum and the neocortex, whereas 30 minutes of ischaemia cause mainly neuronal cell death limited to the striatum. ${ }^{3}$ ( Figure 2) In terms of infarct volume, we expect a standard deviation lower than $30 \%$ in a set of operations. Mortality depends on the occlusion time with around $5 \%$ after $30 \mathrm{~min}$ of ischaemia and $10-20 \%$ after $60 \mathrm{~min}$.

Another minimal invasive possibility is the use of Laser Doppler flowmetry (LDF) during the operation, which allows a direct control of its success In an individual animal, reduction to $10-20 \%$ of preocclusion values clearly indicates successful induction of focal cerebral ischemia. ${ }^{4}$ However, LDF cannot be used as a method for interindividual comparisons, since LDF can only measure quantitative changes (in percentages) of blood flow within a small and limited tissue sample volume. It does not give information about the spatial extent of blood flow reduction.

There are several tests to assess behavioural aspects after stroke, including gait analysis ${ }^{6,7}$, Rotarod ${ }^{8}$, Pole test ${ }^{9,10}$, adhesive removal test 11,12 , staircase test ${ }^{13,14}$, ladder rung test ${ }^{15,16}$ and Morris water maze ${ }^{17}$. In all these tests, mice subjected to MCAo perform less successfully than control animals.

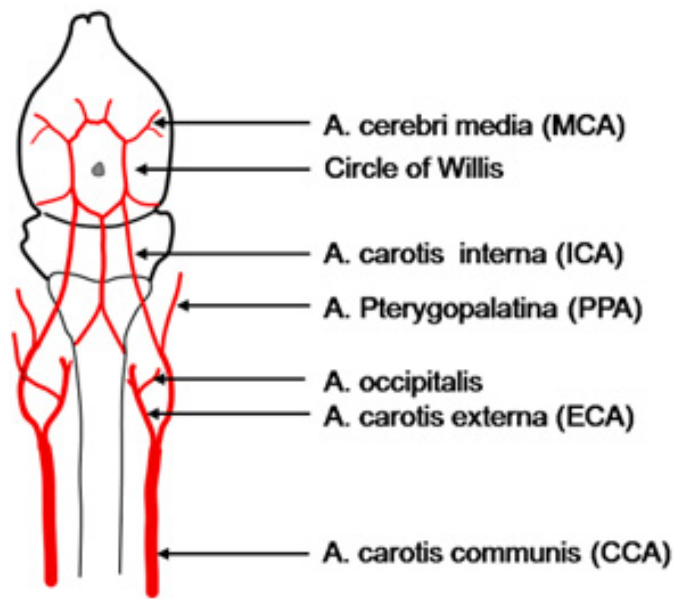

Figure 1. Scheme of the vessel architecture supplying the brain (depicted in background) in the mouse. Different strains may show variations, for example the occipital artery sometimes leaves from the internal carotid artery. 


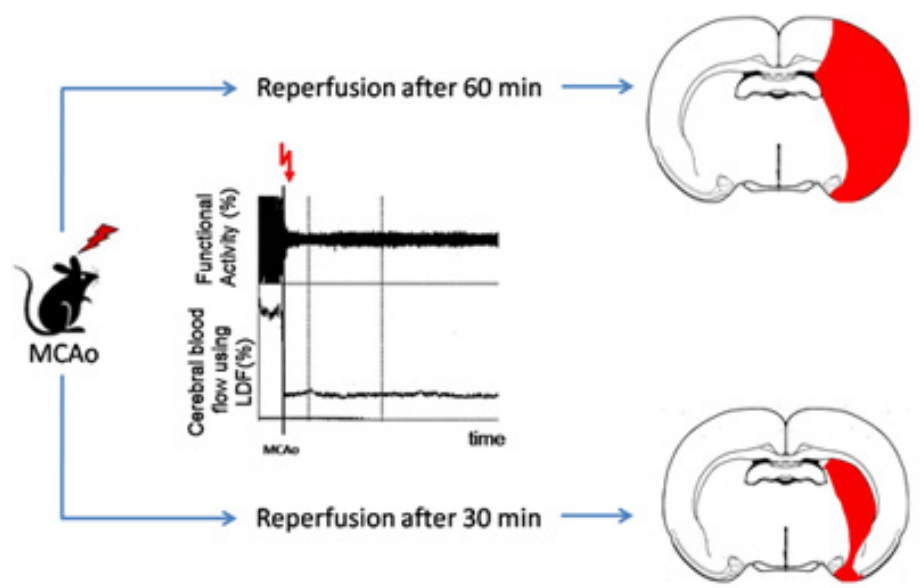

Figure 2. Schematic illustration of typical lesion sizes after different time points of reperfusion in the proximal MCAo model. In the middle, the typical course of functional activity and cerebral blood flow after MCAo are depicted. (MCAo: Middle cerebral artery occlusion, LDF: Laser Doppler flow measurement)

\section{Discussion}

The model of transient, proximal MCA occlusion ${ }^{18,19}$ presented here mimics one of the most common types of ischaemic stroke in patients. ${ }^{20}$ Based on variable reperfusion times, the model offers different grades of damage ranging from transient ischaemic attack (TIA) to large infarcts including major parts of the ischemic hemisphere. This allows the researcher to study different pathophysiological mechanisms after stroke. ${ }^{20,2}$

Surgery can be performed in a short time period and produces highly reproducible lesions. Nevertheless, this requires a thorough control of confounders. ${ }^{22}$ Small differences in operation technique may account for different effects on the infarct. ${ }^{23,24}$ Furthermore, due to variances in cerebral vascular anatomy, different mouse strains show a different outcome. ${ }^{25,26}$ Body temperature affects neurological damage, with hypothermia leading to smaller lesions ${ }^{27}$ and hyperthermia to more severe deficits. ${ }^{28}$ Accordingly, temperature control and maintenance is highly relevant in this model. ${ }^{29}$ In addition, blood pressure and blood gases are important confounders of outcome and need to be monitored. ${ }^{30,31}$ The use of quick and minimal invasive methods (non-invasive blood pressure measurement, suitable and easy accessible blood collection sites) is recommended. The choice of the anaesthetic is also highly important, as some may have neuroprotective effects, and/or be vasodilators, as for example Isoflurane ${ }^{32}$ Consequently, exposure to anaesthesia should be as short as possible and standardized. We exclude animals which have undergone surgery for longer than $15 \mathrm{~min}$.

Shaving the surgical site produces microabrasions and inflammation and releases hair fragments. This may further increase inflammation and foster local infection, which may impact on stroke pathophysiology. Housing conditions, especially the use of enrichment, may affect stroke outcome and should be standardized and described in research reports. ${ }^{6,33,34}$ The use of environmental enrichment and its effect on reproducibility is a matter of discussion. ${ }^{35}$ Another important confounder is the stroke induced risk for infection especially after longer times of ischaemia ${ }^{36}$, which leads to additional morbidity and increased mortality. ${ }^{37,38}$ As infections become symptomatic at around day 3 to 5 , this has important consequences for long term follow-up in such models.

To produce results relevant for the development of novel treatment strategies for stroke, standardization and quality control is highly important in preclinical translational stroke research. ${ }^{39}$ "Good Laboratory Practice" ${ }^{40,41}$ mandates:

1. an appropriate and detailed description of animals used;

2. sample size calculation and reporting on the expected effect size;

3. inclusion and exclusion criteria, set up before the study;

4. randomization of the allocation into groups;

5. allocation concealment with respect to the investigators;

6. reporting animals excluded from analysis;

7. blinded assessment of outcome;

8. reporting potential conflicts of interest and study funding.

\section{Disclosures}

Experiments on animals were performed in accordance with the guidelines and regulations set forth by Landesamt fuer Gesundheit und Soziales, Berlin, Germany.

\section{Acknowledgements}

This work was funded by the European Community's Seventh Framework Programme (FP7/2007-2013) under grant agreement $n^{\circ} 201024$ and $n^{\circ} 202213$ (European Stroke Network). Additional funding was given by the Bundesministerium für Bildung und Forschung (Center for Stroke Research Berlin), and the Deutsche Forschungsgemeinschaft (Exzellenzcluster NEUROCURE). 
The authors would like to thank Mareike Thielke (Dep. for Experimental Neurology, Charité Berlin) for the support during the operation and Elke

Ludwig (Charité Video Services) for producing the animation.

References

1. Dirnagl, U. \& Members of the MCAO-SOP Group, Standard operating procedures (SOP) in experimental stroke research: SOP for middle cerebral artery occlusion in the mouse. Nature Precedings (Available online <http://precedings.nature.com/documents/3492/version/2>) (2010).

2. Bederson, J.B. et al., Rat middle cerebral artery occlusion: evaluation of the model and development of a neurologic examination. Stroke 17 (3), 472-476 (1986).

3. Katchanov, J. et al., Selective neuronal vulnerability following mild focal brain ischemia in the mouse. Brain Pathol 13 (4), $452-464$ (2003).

4. Dirnagl, U., Complexities, Confounders, and Challenges in Experimental Stroke Research: A checklist for researchers and reviewers in Rodent models of stroke, edited by U. Dirnagl (Springer, New York Dordrecht Heidelberg London, 2010), pp. 267.

5. Dirnagl, U., Kaplan, B., Jacewicz, M., \& Pulsinelli, W., Continuous measurement of cerebral cortical blood flow by laser-Doppler flowmetry in a rat stroke model. J Cereb Blood Flow Metab 9 (5), 589-596 (1989).

6. Wang, Y. et al., A comprehensive analysis of gait impairment after experimental stroke and the therapeutic effect of environmental enrichment in rats. J Cereb Blood Flow Metab 28 (12), 1936-1950 (2008).

7. Lubjuhn, J. et al., Functional testing in a mouse stroke model induced by occlusion of the distal middle cerebral artery. $J$ Neurosci Methods $184(1), 95-103$ (2009).

8. Jones, B.J. \& Roberts, D.J., The quantiative measurement of motor inco-ordination in naive mice using an acelerating rotarod. J Pharm Pharmacol 20 (4), 302-304 (1968).

9. Matsuura, K., Kabuto, H., Makino, H., \& Ogawa, N., Pole test is a useful method for evaluating the mouse movement disorder caused by striatal dopamine depletion. J Neurosci Methods 73 (1), 45-48 (1997).

10. Bouet, V. et al., Sensorimotor and cognitive deficits after transient middle cerebral artery occlusion in the mouse. Exp Neurol 203 (2), $555-567$ (2007).

11. Freret, T. et al., Delayed administration of deferoxamine reduces brain damage and promotes functional recovery after transient focal cerebral ischemia in the rat. Eur J Neurosci 23 (7), 1757-1765 (2006).

12. Modo, M. et al., Neurological sequelae and long-term behavioural assessment of rats with transient middle cerebral artery occlusion. $J$ Neurosci Methods 104 (1), 99-109 (2000).

13. Montoya, C.P., Campbell-Hope, L.J., Pemberton, K.D., \& Dunnett, S.B., The "staircase test": a measure of independent forelimb reaching and grasping abilities in rats. $J$ Neurosci Methods 36 (2-3), 219-228 (1991).

14. Baird, A.L., Meldrum, A., \& Dunnett, S.B., The staircase test of skilled reaching in mice. Brain Res Bull 54 (2), $243-250$ (2001).

15. Metz, G.A. \& Whishaw, I.Q., Cortical and subcortical lesions impair skilled walking in the ladder rung walking test: a new task to evaluate foreand hindlimb stepping, placing, and co-ordination. J Neurosci Methods 115 (2), 169-179 (2002).

16. Farr, T.D., Liu, L., Colwell, K.L., Whishaw, I.Q., \& Metz, G.A., Bilateral alteration in stepping pattern after unilateral motor cortex injury: a new test strategy for analysis of skilled limb movements in neurological mouse models. J Neurosci Methods 153 (1), 104-113 (2006).

17. Morris, R., Developments of a water-maze procedure for studying spatial learning in the rat. J Neurosci Methods 11 (1), 47-60 (1984).

18. Koizumi, J., Yoshida, Y., Nakazawa, T., \& Ooneda, G., Experimental studies of ischemic brain edema. I: a new experimental model of cerebral embolism in rats in which recirculation can be introduced in the ischemic area. Jpn J Stroke 8, 1 - 8 (1986).

19. Longa, E.Z., Weinstein, P.R., Carlson, S., \& Cummins, R., Reversible middle cerebral artery occlusion without craniectomy in rats. Stroke 20 (1), 84-91 (1989)

20. Endres, M. \& Dirnagl, U., Neuroprotective Strategies in Animal and in vitro-Models of Neuronal Damage: Ischemia and Stroke in Molecular and Cellular Biology of Neuroprotection in the CNS, edited by Christian Alzheimer (Kluver Academic and Landes Bioscience, New York, 2003), Vol. 513, pp. 455 - 474.

21. Dirnagl, U., ladecola, C., \& Moskowitz, M.A., Pathobiology of ischaemic stroke: an integrated view. Trends Neurosci 22 (9), $391-397$ (1999).

22. Liu, S., Zhen, G., Meloni, B.P., Campbell, K., \& Winn, H.R., Rodent Stroke Model Guidelines for Preclinical Stroke Trials (1st Edition). J Exp Stroke Transl Med 2 (2), 2-27 (2009).

23. Chen, Y., Ito, A., Takai, K., \& Saito, N., Blocking pterygopalatine arterial blood flow decreases infarct volume variability in a mouse model of intraluminal suture middle cerebral artery occlusion. J Neurosci Methods 174 (1), 18-24 (2008).

24. Tsuchiya, D., Hong, S., Kayama, T., Panter, S.S., \& Weinstein, P.R., Effect of suture size and carotid clip application upon blood flow and infarct volume after permanent and temporary middle cerebral artery occlusion in mice. Brain Res 970 (1-2), 131-139 (2003).

25. Beckmann, N., High resolution magnetic resonance angiography non-invasively reveals mouse strain differences in the cerebrovascular anatomy in vivo. Magn Reson Med 44 (2), 252-258 (2000).

26. Barone, F.C., Knudsen, D.J., Nelson, A.H., Feuerstein, G.Z., \& Willette, R.N., Mouse strain differences in susceptibility to cerebral ischemia are related to cerebral vascular anatomy. J Cereb Blood Flow Metab 13 (4), 683-692 (1993).

27. Florian, B. et al., Long-term hypothermia reduces infarct volume in aged rats after focal ischemia. Neurosci Lett 438 (2), $180-185$ (2008).

28. Noor, R., Wang, C.X., \& Shuaib, A., Effects of hyperthermia on infarct volume in focal embolic model of cerebral ischemia in rats. Neurosci Lett 349 (2), 130-132 (2003).

29. Barber, P.A., Hoyte, L., Colbourne, F., \& Buchan, A.M., Temperature-regulated model of focal ischemia in the mouse: a study with histopathological and behavioral outcomes. Stroke 35 (7), 1720-1725 (2004)

30. Shin, H.K. et al., Mild induced hypertension improves blood flow and oxygen metabolism in transient focal cerebral ischemia. Stroke 39 (5), 1548-1555 (2008).

31. Bottiger, B.W. et al., Global cerebral ischemia due to cardiocirculatory arrest in mice causes neuronal degeneration and early induction of transcription factor genes in the hippocampus. Brain Res Mol Brain Res 65 (2), 135-142 (1999).

32. Kapinya, K.J., Prass, K., \& Dirnagl, U., Isoflurane induced prolonged protection against cerebral ischemia in mice: a redox sensitive mechanism? Neuroreport 13 (11), 1431-1435 (2002).

33. Endres, M. et al., Mechanisms of stroke protection by physical activity. Ann Neurol 54 (5), 582-590 (2003). 
34. Gertz, K. et al., Physical activity improves long-term stroke outcome via endothelial nitric oxide synthase-dependent augmentation of neovascularization and cerebral blood flow. Circ Res 99 (10), 1132-1140 (2006).

35. Richter, S.H., Garner, J.P., \& Wurbel, H., Environmental standardization: cure or cause of poor reproducibility in animal experiments? Nat Methods 6 (4), 257-261 (2009).

36. Liesz, A. et al., The spectrum of systemic immune alterations after murine focal ischemia: immunodepression versus immunomodulation. Stroke 40 (8), 2849-2858 (2009).

37. Meisel, C., Schwab, J.M., Prass, K., Meisel, A., \& Dirnagl, U., Central nervous system injury-induced immune deficiency syndrome. Nat Rev Neurosci 6 (10), 775-786 (2005).

38. Engel, O. \& Meisel, A., Models of Infection Before and After Stroke: Investigating New Targets. Infect Disord Drug Targets 9 (5) (2010).

39. Dirnagl, U., Bench to bedside: the quest for quality in experimental stroke research. J Cereb Blood Flow Metab 26 (12), 1465-1478 (2006).

40. Macleod, M.R. et al., Good laboratory practice: preventing introduction of bias at the bench. Stroke 40 (3), e50-52 (2009).

41. STAIR Group, Recommendations for standards regarding preclinical neuroprotective and restorative drug development. Stroke 30 (12), 2752-2758 (1999). 